\title{
Fibronectin Absorbed onto TiN-coated and Uncoated Titanium Alloy Implants Enhances Osseointegration in the Rat Femur.
}

\author{
A. Weiss. L, G. Sovak, ${ }^{*}$ and I. Gotman ${ }^{* *}$ \\ *Department of Anatomy \& Cell Biology, The Rappaport Faculty of Medicine, Technion_Israel \\ Institute of Technology, P.O.Box 9649, Haifa 31096, Israel \\ ** Department of Materials Engineering, Technion, Haifa, Israel
}

The use of uncemented endosseous implants in restorative dentistry and orthopedics is increasing steadily. Titanium and its alloys, in particular Ti6Al4V, are the materials of choice for such implants. Titanium is characterized by an excellent capacity for osseointegration, has a superior corrosion resistance, high fatigue strength and low elastic modulus. However, the disadvantage of both pure titanium and Ti6Al4V is their relatively poor wear resistance. Hard TiN ceramic coatings may improve the relatively poor wear-resistance and increase the life-span of Ti-based implants. To improve TiN adhesion, an original PIRAC coating method, in which a TiN coating is formed by the interaction of a Ti-based substrate with monatomic nitrogen, has been developed [1]. The novel TiN coating was found to be highly biocompatible and demonstrated osseointegration capacity comparable to that of titanium alloy itself [2].

Biological fixation of endosseous implants requires apposition of bone on implant's surface, in a process named osseointegration. Osseointegration progresses slowly, and requires long periods of immobilization or unloading. Fibronectin is an abundant extracellular matrix component and is recognized by osteoblasts through integrin receptors [3]. It has been shown that the recognition of RGD by integrins is responsible for the adhesion of osteoblast-like cells to fibronectin coated surfaces in vitro [4], and RGD coated titanium implants in vivo [5].

The aim of this research was to test the effect of fibronectin on early osseointegration of titanium alloy implants. Tin-coated or uncoated (control) Ti-6Al-4V pins were preabsorbed with fibronectin, and were implanted in the distal femurs of 6-month-old female Wistar rats. Animals were sacrificed 10 days after implantation. To demonstrate mineralization, animals received a single dose of oxytetracyclin, 3 days before sacrificed. Femurs were embedded undecalcified and examined by scanning electrone microscope (SEM). Morphometric analyses were performed with the aid of ImagePro software, as described previously [2]. SEM revealed that within the distal epiphyses the implants were embedded in a trabecular bone. Bone volume was measured within a distance of $0.5 \mathrm{~mm}$ from implants' surface and revealed a significant increase with the fibronectin-precoated implants in comparison to control implants (Table 1). In the femoral diaphyses, at 10 days after implantation, no bone can be seen in the animals into which pins without fibronectin were inserted (Fig. 1A,C), yet it is filled with trabecular bone in animals implanted with pins onto which fibronectin was adsorbed, (Fig. 1B,D; Table 1).

Tetracyclin labeling indicated an increase in mineralization around the fibronectin-coated implants (not shown). Immunohistochemical labeling revealed that fibronectin is expressed in the bone surrounding control implants, yet its expression is significantly increased with the fibronectin-coated implants. In addition, alkaline phosphatase activity was significantly increased with fibronectin precoated implants.

In conclusion, our findings indicate that coating implants with fibronectin, stimulates bone formation around titanium alloy implants, both TiN-coated or uncoated and accelerates their osseointegration, probably due to its ability to promote osteoblasts adhesion to the biomaterial. 


\section{References}

[1] A . Shenhar, I. Gotman, S. Radin, P. Ducheyne and E.Y. Gutmanas, Surface Coatings Technol 126 (2000) 210.

[2] Sovak G, Weiss A, Gotman I.. J Bone Joint Surg [Br];82-B (2000):290.

[3] Akiyama SK. Hum Cell 9 (1996):181.

[4] Degasne I. et al. Calcif Tissue Int 64 (1999):499.

[5] Hennessy KM. et al. Biomat 29 (2008):3075.

Table 1. Effect of fibronectin on bone volume and bone-implant contact. 10 days after implantation.

\begin{tabular}{|c|c|c|c|c|}
\hline & \multicolumn{2}{|c|}{$\underline{B V(\%)}$} & \multicolumn{2}{|c|}{ BIC (\%) } \\
\hline & TiN-coated & uncoated & TiN-coated & uncoated \\
\hline \multirow{2}{*}{$\begin{array}{l}\text { Control } \\
\text { Epiphysis } \\
\text { Diaphysis }\end{array}$} & $47.3 \pm 4.3$ & $42.5 \pm 4.0$ & $82.5 \pm 3.3^{*}$ & $84.3 \pm 4.7^{*}$ \\
\hline & - & - & $4.3 \pm 1.1$ & $3.1 \pm 1.0$ \\
\hline \multirow{2}{*}{$\begin{array}{l}\text { Fibronectin } \\
\text { Epiphysis } \\
\text { Diaphysis }\end{array}$} & $63.8 \pm 3.0 *$ & $61.6 \pm 3.5^{*}$ & $81.6 \pm 4.8^{*}$ & $72.4 \pm 2.2 *$ \\
\hline & - & - & $41.8 \pm 10.5$ & $30.6 \pm 5.1$ \\
\hline
\end{tabular}

BV and BIC were measured on SEM images with ImagePro. *, $\mathrm{p}<0.05$ in comparison to control by ANOVA. The differences between TiN-coated and uncoated pins were non-significant.
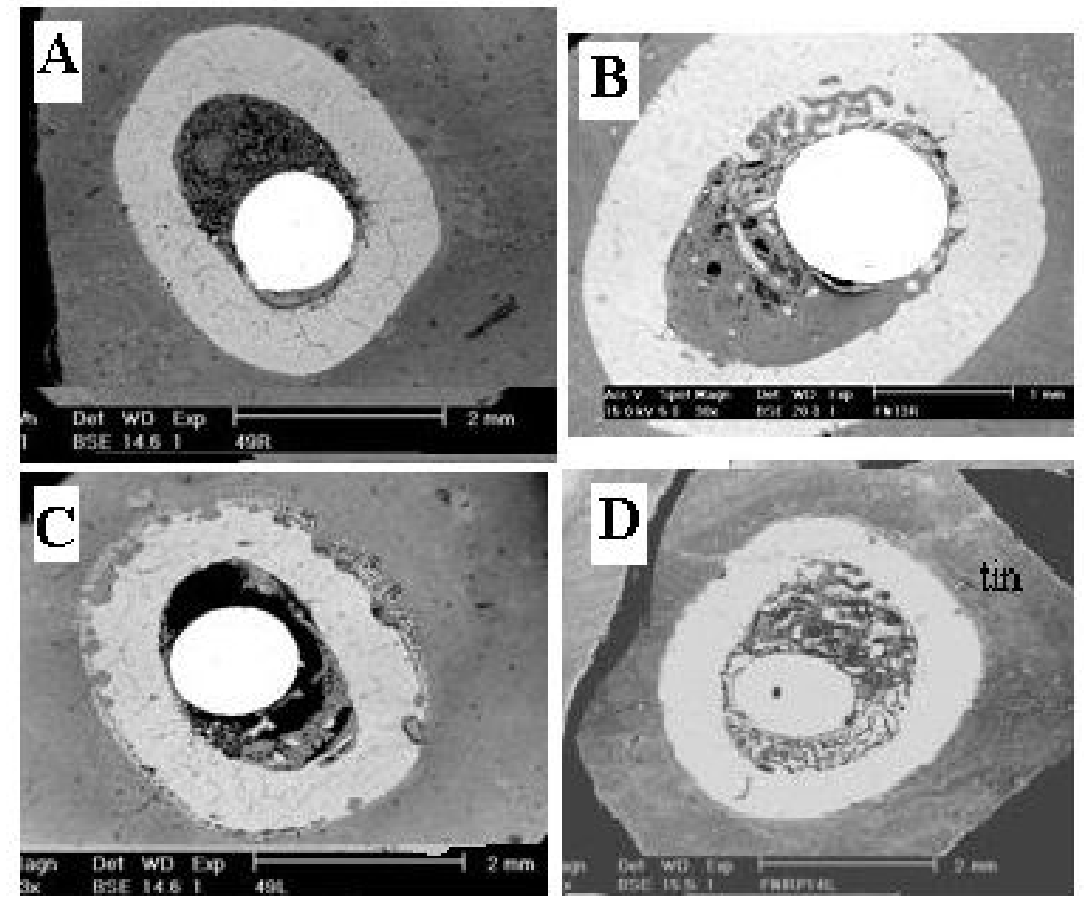

Fig. 1. SEM images of transverse sections of rat femoral diaiphyses, 10 days after implantation: A, uncoated implant; B, uncoated implant with fibronectin; C, TiN-coated implant; D, TiN-coated implant with fibronectin. 